\title{
An Audit Evaluating Local Compliance with NICE NG12 Suspected Cancer: Recognition and Referral Pathway at Bridgewater NHS Foundation Trust Community Dental Services
}

\section{Iffah Zaman* and Zara Leighton}

Dental Officer, Community Dental Services, Bridgewater NHS Foundation Trust, United Kingdom

*Corresponding Author: Iffah Zaman, Dental Officer, Community Dental Services, Bridgewater NHS Foundation Trust, United Kingdom.
Received: December 15, 2021

Published: January 06, 2022

(C) All rights are reserved by Iffah Zaman and

Zara Leighton.

\begin{abstract}
Background: Dentists have a responsibility to thoroughly assess the head and neck area for patients during clinical visits regardless of the patients presenting issue. This enables dental practitioners to help identify patients with signs or symptoms that could be caused by cancer.

Aims: This Audit aims to evaluate local compliance in a Primary Care setting with Nice Guidelines (NG12) for suspected cancer: recognition and referral pathway across the Community Dental Services at Bridgewater NHS Foundation Trust at the Oldham, Rochdale and Bury Boroughs (ORB).

Methods: This retrospective audit examined a sample of 30 urgent referrals made across the ORB boroughs from January 2019 to May 2020 for compliance with national NG12 guidelines.

Results: Results showed that none of the patients met $100 \%$ of the criteria for suspected cancer referrals. $13.3 \%$ of patients that were referred had confirmed cancer that we are aware of. $100 \%$ of referrals were sent due to signs/symptoms that conform to the NICE NG12 guidelines. There was 100\% compliance with practitioners documenting patient symptoms. 10\% of patients had no mention of their cancer risk factors in their records.

$13 \%$ of patients had no documented consent for their urgent referral. $37 \%$ of patients had no clinical documentation of any further information/support provided to them. $13.3 \%$ of patients who had suspected cancer referrals sent were followed up by us within 2 weeks. For $33.3 \%$ of the patients we referred we never heard back from the specialist department.

Conclusion: We should address the inconsistencies identified with clinical record keeping, addressing risk factors, gaining valid consent and the follow-up process both locally and from specialist services.
\end{abstract}

Keywords: Audit; Suspected Cancer; NG12; NICE; Community Dental Services

\section{Abbreviations}

NICE NG12: The National Institute for Health and Care Excellence Guidelines; KPI: Key Performance Indicator; R4: CS R4+ Practice Management Software; BDA: British Dental Association

\section{Introduction}

\section{Background}

Dentists have a responsibility to thoroughly assess the head and neck area for patients during clinical visits regardless of the patients presenting issue. Not only is this assessment process an

Citation: Iffah Zaman and Zara Leighton. "An Audit Evaluating Local Compliance with NICE NG12 Suspected Cancer: Recognition and Referral Pathway at Bridgewater NHS Foundation Trust Community Dental Services". Acta Scientific Dental Sciences 6.2 (2022): 31-36. 
important prerequisite for carrying out necessary dental treatment but it also enables dental practitioners to help identify patients with signs or symptoms that could be caused by cancer [2].

Aims

- To evaluate local compliance in a Primary Care setting with Nice Guidelines (NG12) for suspected cancer: recognition and referral pathway across the Community Dental Services at Bridgewater NHS Foundation Trust (across the "ORB" Oldham, Rochdale and Bury Boroughs).

- To identify potential areas for improvement, implement changes to improve compliance with current guidelines and eventually re-audit to evaluate changes.

\section{Objectives}

- $\quad$ Evaluate whether current local ORB practices are in line with national NICE guidelines for recognising and referring signs/ symptoms that could be caused by cancer

- Retrospectively assess whether appropriate primary investigations and records are being made; including appropriate intra and extra oral assessments for suspicious features prior to sending an urgent referral.

- Determine whether urgent 2 week wait referrals that have been made to specialists in secondary care are suitable.

- To assess the record keeping and consent procedures for urgent referrals compared to NICE guidelines

- To examine current practices for supporting patients if they have signs/symptoms that may suggest cancer and providing appropriate further support.

\section{Materials and Methods}

Methodology

- This is a retrospective, cross-sectional, records-based audit which examined urgent referrals made across the ORB boroughs following the urgent 2 week referral process.

- The referrals which were sent to the appropriate secondary care specialist centre were analysed for compliance with national NG12 guidelines [1].

- This Audit assessed our compliance with the NG12 guidelines and identified areas for improvement [1].

\section{Sample}

A sample of 30 urgent referrals made across the ORB boroughs following the urgent 2 week referral process for suspected cancer patients from January 2019 to May 2020 were analysed.

\section{Data source}

The data was sourced from patient records on the "R4" Dental Software used for record keeping across the ORB boroughs. This also included letters which were scanned onto the R4 software. We also accessed patient referrals directly from the Greater Manchester National Urgent 2 week wait referral site: https://www.dentalreferrals.org/dentists/.

\section{Data collection methods}

A spreadsheet was designed with the following points $[2,3]$ :

- $\quad$ Patient R4 identifier (R4 dental software for record keeping)

- $\quad$ Referral number

- $\quad$ Patient age and sex

- $\quad$ Clinic location

- Date of assessment

- Were correct patient details included on the referral?

- Were correct details of the referring practitioner and clinic included on the referral?

- Were the correct details for the specialist/department included on the referral?

- Was the area of suspicion documented?

- Do the suspicious signs meet NICE criteria?

- Were patient symptoms documented?

- Were risk factors discussed and documented?

- How were the risk factors documented

- Was the urgent referral sent within 24 hours?

- Was patient consented for urgent referral?

- Was patient followed up to ensure they received an appointment within 2 weeks?

- Was the patient seen in urgent dental care?

- Was the patient given further information for support available?

- If the patient was given further information for support, did it include a) why/where they are being referred, b) how long 
to expect to wait for the appointment, c) what type of tests may be carried out, d) who to contact for further information?

- $\quad$ Did the assessment and referral process meet all of the criteria listed in this spreadsheet?

\section{Limitations}

A potential limitation of this Audit is that the sample was for only 30 patients. Future re-audit may consider a larger sample. Data collection was cross-checked by the two clinicians undertaking the audit (I Zaman and Z Leighton).

\section{Standards derived from}

National

- Suspected cancer: recognition and referral NICE guideline (NG12) Published 20/06/2015

- $\quad$ GDC Standards for Dental Professionals

- $\quad$ FGDP: Standards in Dentistry

- FGDP: Clinical examination and record keeping; good practice guidelines.

\section{Regional}

GM National Urgent 2WW cancer online referral form: operated by FDS Consultant and accessed via the https://www.dental-referrals.org/dentists/portal.

Local

Bridgewater Community Healthcare NHS Foundation Trust policies:

- Health Records Policy

- $\quad$ Records Management - Storing and Movement of Records Policy

- $\quad$ Records Management Archiving Retention Disposal Policy.

\section{Results and Discussion}

\section{Patient demographics}

- We analysed records and referrals for 30 patients. This included 24 male patients and 6 female patients.

- $\quad$ The average patient age was 54.8 ranging from 15 - 74 years.

- $\quad$ These referrals were made between 10/1/19-18/5/2020.

- The indication for the referral for one patient from $18 / 05 / 2020$ was actually initially recognised by the clinician on 24/04/2019 but there was substantial delay in sending the referral due to the patient declining to consent. Therefore, this referral was only sent on 18/05/2020.

- $\quad 2 / 30(6.7 \%)$ of the referrals were made during the covid-19 pandemic lockdown on $17 / 3 / 20$ and 18/5/20.

- $\quad 23 / 30$ patients (76.7\%) referred with suspected cancer were urgent dental care patients

- $\quad$ For $4 / 30$ (13.3\%) patients that were referred had cancer was detected (this number may be higher as 10/30 (33.3\%) had no letter response from specialist services).

\section{Accuracy of referral details}

- $30 / 30(100 \%)$ of referrals contained the correct patient details.

- $\quad 2 / 3(66.75 \%)$ of the referrals had the wrong clinician address listed. All of the electronic referrals on the portal appear to default to the Bury clinic as the clinic site regardless of where the patient was actually seen.

Do the suspicious signs meet NICE criteria?

The NICE NG12 guidelines mention the following criteria for suspicious signs (Figure 1).

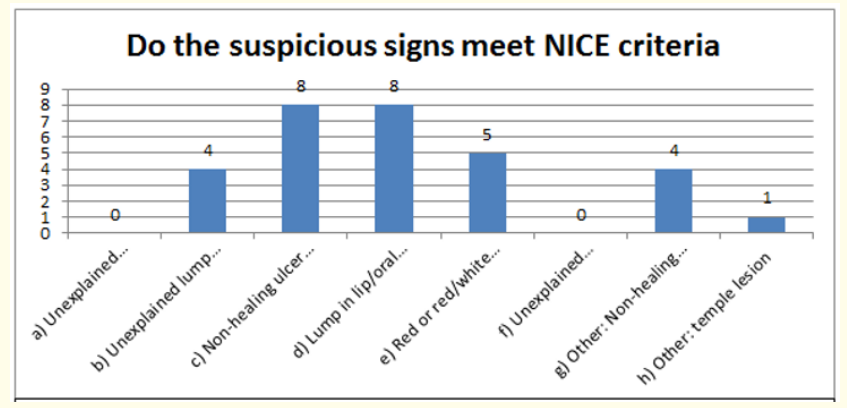

Figure 1: Do the suspicious signs meet NICE criteria?

a. Unexplained hoarseness

b. Unexplained lump in neck

c. Non healing ulcer $>3$ weeks

d. Lump in lip/oral cavity

e. Red or red/white patch in oral cavity

f. Unexplained thyroid lump

g. Other: Non-healing socket

h. Other: temple lesion 
4/30 (13.3\%) of referrals were sent for a "non-healing socket". A non-healing socket is not necessarily listed in the NICE guidelines as a suspicious sign, but depending on the individual case this may require urgent referral. 25/30 (83.3\%) of referrals did meet specific NICE criteria for suspicious signs/symptoms. $1 / 30$ patients was referred for a temple lesion.

\section{Patient symptoms}

There was $100 \%$ compliance with practitioners documenting patient symptoms. For $28 / 30$ (93.3\%) patients the symptoms were documented both on R4 clinical software and in the urgent referral form. The remaining 2 patients had their symptoms documented on R4 software and not in the referral.

\section{Risk factors}

Figure 2 below illustrates how clinicians are recording oral cancer risk factors such as smoking, alcohol consumption and previous cancer diagnosis $[2,3]$. As can be seen there is no consistent way that clinicians appear to be doing this.

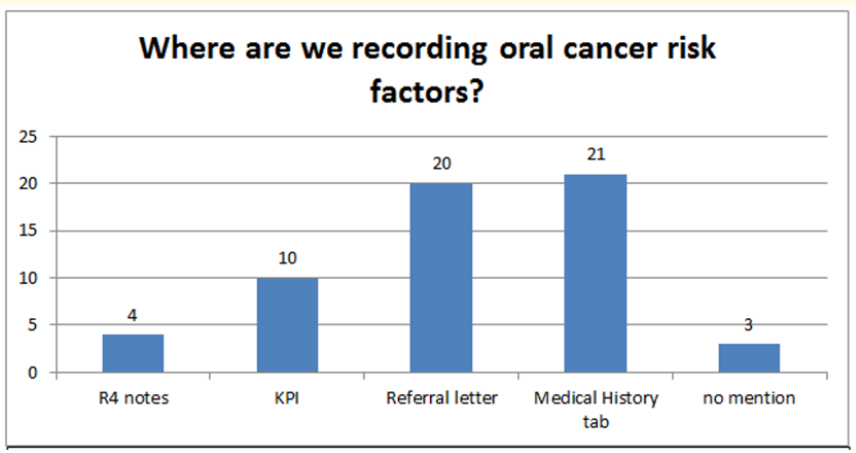

Figure 2: Distribution of methods used by clinicians for recording risk factors for oral cancer.

Further to the above, 2 patients had conflicting information recorded for their risk factors.

A patient had the KPI (key performance indicator) ticked for smoking cessation in their treatment plan but the patient was recorded as a non-smoker in their medical history.

Another patient was recorded as an ex-smoker in their clinical records but did not have this risk factor recorded on their referral [2].
Time taken to refer

There was $96.7 \%$ compliance $(29 / 30)$ in sending referrals within 24 hours.

One patient would not consent to his referral being sent despite the clinician explaining the urgency and reason. The patients also would not agree to going to any specialist appointments without sedation/private transfer which caused substantial delay. This highlights potential patient management challenges especially in Community Dental Services.

\section{Consent for referral}

Figure 3 below shows the various ways that clinicians were recording the consent process for urgent referrals.

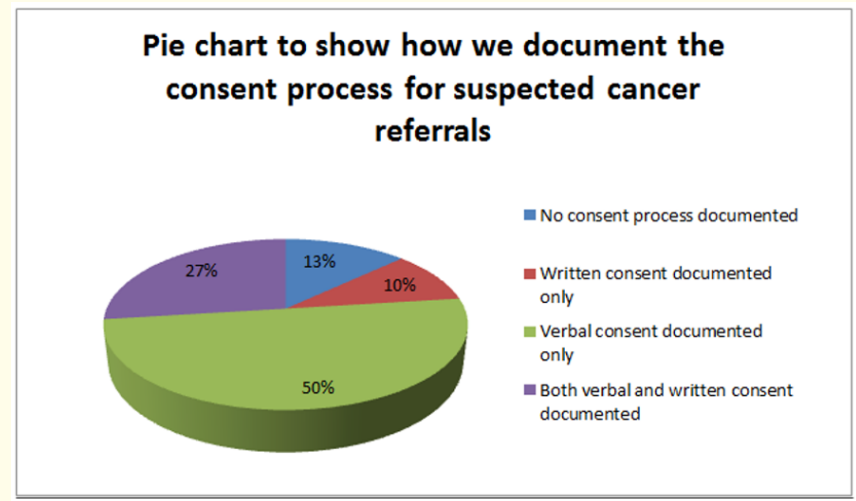

Figure 3: Chart showing documentation for the consent process for suspected cancer referrals.

The quality of written consent was varied, for example some written consent forms only stating to "check tongue" and "referral to hospital".

For one patient the clinician had written "consent not required".

The quality of documentation for verbal consent varied greatly too within the clinical records. Many clinicians appear to be using the generic routine exam template for suspected cancer patients too. Documentation included phrases such as "verbal consent gained" but was not specific for the importance of the referral.

Further information given to patient with regards to referral

Table 1 below illustrates the various ways clinicians are documenting the provision of further information to patients after informing them of the need to make a suspected cancer referral. 


\begin{tabular}{|l|c|}
\hline $\begin{array}{l}\text { How many patients had documentation of } \\
\text { further information given }\end{array}$ & $\begin{array}{c}\text { Number of } \\
\text { patients }\end{array}$ \\
\hline Why/where they are being referred to & $16(53 \%)$ \\
\hline How long to expect to wait for the appointment & $13(43 \%)$ \\
\hline What type of tests may be carried out & $3(10 \%)$ \\
\hline Who to contact for further information & $3(10 \%)$ \\
\hline
\end{tabular}

Table 1

As per the previous observations, it appears that for further information and support, there is no consistent method of delivering this to patients. Most of the advice was given verbally and documented in the clinical records.

Additionally, $11 / 30$ patients (37\%) had no documentation of what information was given or further support.

Follow up

- $\quad 4 / 30$ patients $(13.3 \%)$ who had suspected cancer referrals sent were followed up by us within 2 weeks. For another patient the follow-up was 4 weeks after the referral and appears to be a coincidence as the patient happened to be back for a visit after 4 weeks to complete other treatment.

- $\quad$ All other patients $25 / 30(83.3 \%)$ had no documented attempt to check that a specialist appointment had been attended or made. This follow-up process may be even more relevant in Community Dental Service as we deal with many more vulnerable patients and the follow-up can be complicated.

\section{Follow up letters}

Figure 4 below illustrates the distribution of the number of responses received from specialist services after a suspected cancer referral was sent.

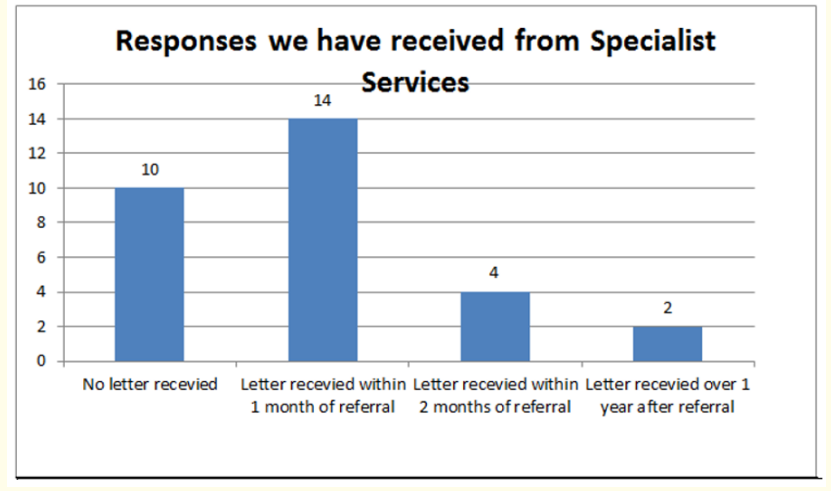

Figure 4: Responses from specialist services for patients with suspected cancer referrals (number of patients).
$10 / 30$ patients (33.3\%) had no documented response on the R4 software from specialist services after the suspected cancer referral was sent to specialist services.

For most of the other responses we received from specialist services, there seems to be a great variation in acknowledging the letters in the notes. Sometimes we base the outcome purely on the patients' feedback to us without any letter from hospital.

Clinicians also rely on administrative staff to let them know if any letters have arrived. There appears to be no set protocol for notifying clinicians of received letters.

\section{Discussion}

None of the patients met $100 \%$ of the NG12 criteria for suspected cancer referrals. Therefore changes should be made to make this process more effective.

The importance of suspected cancer referrals being sent in a timely manner is highlighted by the fact that $4 / 30(13.3 \%)$ of patients that were referred had confirmed cancer that we are aware of [1]. The level of response from specialist services was low therefore this figure may be higher.

One patient was initially "diagnosed" with potentially cancerous signs by the clinician on 24/04/2019. The patient had capacity and the clinician documented clearly that the signs appear indicative of cancer [2]. Despite other 2 week wait referrals being sent for this patient in the meantime, the patient refused to attend the hospital for these appointments. Therefore, the 2 week wait referral included in this audit for this patient was not sent until 18/05/2020 due to lack of consent. This patient was eventually confirmed to have a Squamous Cell Carcinoma. This shows the challenges clinicians may face when treating vulnerable patients in community services and highlights the importance of follow up and ensuring attendance to hospital appointments [3].

Overall record keeping doesn't appear to always be consistent or accurate regarding suspected cancer patients especially when documenting risk factors [2]. This may relate to staff training and knowledge or perceived time restrictions.

The quality of verbal and written consent was varied, for example some written consent forms stated the patient was being referred "to check tongue" and "referral to hospital". It is important that patients understand why they are being referred to help ensure attendance for the hospital appointment and ensure valid 
consent. Reasons for not explaining findings clearly to patients may relate to fear over worrying the patient.

Only 4/30 (13.3\%) of patients who had suspected cancer referrals sent had documented follow up with us, which does not meet standards.

The fact that $23 / 30(77 \%)$ of the patients in this audit were urgent dental care patients may likely affect the follow up process and results because these patients are seen as a one off emergency.

\section{Conclusion}

We should address the inconsistencies identified with clinical record keeping, addressing risk factors, gaining valid consent and the follow-up process both locally and from specialist services [1]. This will improve the urgent cancer pathway for patients and also help clinicians to provide appropriate care.

\section{Recommendations}

Sharing and disseminating the audit results locally will be informative and helpful for the boroughs and the wider Bridgewater dental team.

We would benefit from a centralised way of logging all of the suspected cancer referrals sent out by the department.

As the ORB dental services have merged with Bridgewater NHS Trust there are now existing patient information leaflets regarding the 2 week referrals which clinicians can use.

We would benefit from a standardised template for documenting notes on R4 software for urgent 2 week wait referrals which would also help to standardise the health promotion, cessation advice, discussion of risk factors and consent process.

As evidenced by the results, the risk factors for oral cancer can be documented in several different ways e.g. the notes, medical history, on KPI's (key performance indicators) or on the referral itself. We would benefit from having a standardised system to document risk factors such as a template, which would also help to identify high risk patients.

A standard template would help to remind clinicians to document details such as number of cigarettes smoked per day/for how long and units of alcohol consumption which isn't being recorded consistently at the moment.
Smoking cessation and alcohol advice leaflets would also be helpful if added to the communications section on R4 software so that when they are given to patients there is an automatic trace that the letters were given.

We would encourage clinicians to utilise the "BDA-CRUK Oral Cancer Recognition Toolkit" which is designed to improve our understanding of the prevention and detection of oral cancer and how to respond effectively.

\section{Conflict of Interest}

None.

\section{Bibliography}

1. Nice.org.uk. Overview. "Suspected Cancer: Recognition And Referral”. Guidance | NICE (2020).

2. Cancerresearchuk.org. "Risks And Causes". Mouth Cancer | Cancer Research UK (2020).

3. Greater Manchester Combined Authority (GMCA). Oral Cancer Care- prevention, early detection, referral, care. Local Guide advice and guidance for the primary care dental team. $1^{\text {st }}$ Edition (2019).

\section{Assets from publication with us}

- Prompt Acknowledgement after receiving the article

- Thorough Double blinded peer review

- Rapid Publication

- Issue of Publication Certificate

- High visibility of your Published work

Website: www.actascientific.com/

Submit Article: www.actascientific.com/submission.php

Email us: editor@actascientific.com

Contact us: +919182824667 